\title{
The homopurine-homopyrimidine DNA sequence flanking the thyroglobulin gene does not behave as a transcriptional diode in living cells
}

This article was published in the following Dove Press journal:

Research and Reports in Biology

27 July 2015

Number of times this article has been viewed

\section{Christiane Christophe- Hobertus Daniel Christophe}

Institut de Recherche Interdisciplinaire en Biologie Humaine et Moléculaire (IRIBHM), Institut de Biologie et de Médecine Moléculaires (IBMM), Faculté de Médecine, Université Libre de Bruxelles, Gosselies, Belgium
Correspondence: Daniel Christophe Institut de Recherche Interdisciplinaire en Biologie Humaine et Moléculaire (IRIBHM), Institut de Biologie et de Médecine Moléculaires (IBMM), rue des Professeurs Jeeners et Brachet 12, B-604I Gosselies, Belgium

$\mathrm{Tel}+3226509828$

Fax +32 26509820

Email dchristo@ulb.ac.be
Abstract: Homopurine-homopyrimidine DNA sequences are found at higher than expected frequency around gene promoters in mammalian genomes. They were reported to act as a transcriptional diode in in vitro assays, as only the homopurine DNA strand was transcribed. We show here that the homopurine-homopyrimidine upstream element of the thyroglobulin gene promoter is able to reduce transcription initiated from different promoters, both on transiently transfected plasmids or when integrated into the genome of rat thyroid cells. In living cells, this homopurine-homopyrimidine sequence inhibits transcription of both DNA strands.

Keywords: $\mathrm{Pu} / \mathrm{Py}$, promoter, transcription, thyroglobulin, thyroid

\section{Introduction}

Long homopurine-homopyrimidine $(\mathrm{Pu} / \mathrm{Py}) \mathrm{DNA}$ sequences are found in mammalian genomes at much higher frequency than that estimated by chance alone, raising lots of speculation about their possible function(s). ${ }^{1} \mathrm{Pu} / \mathrm{Py}$ DNA motifs are also found at higher than expected frequency around transcription units, ${ }^{2}$ and in many instances in the upstream region. ${ }^{3}$ Some of these sequences are localized just next to, or within, gene promoters and have been reported to play a role in the control of promoter strength in a few cases. ${ }^{4,5} \mathrm{~A}$ few have even been reported to display intrinsic promoter function. ${ }^{6}$ DNA-binding proteins have also been shown to specifically recognize $\mathrm{Pu} / \mathrm{Py}$ stretches and to behave as transcriptional regulators in some instances. ${ }^{7} \mathrm{Pu} / \mathrm{Py} \mathrm{DNA}$ sequences were also reported to reduce DNA replication ${ }^{8-10}$ and to favor DNA recombination. ${ }^{11,12}$ In appropriate conditions, repeat-containing $\mathrm{Pu} / \mathrm{Py} \mathrm{DNA}$ sequences are known to adopt a triple-helical structure called H-DNA. ${ }^{13,14}$

Regarding DNA transcription, it has been shown $n^{3,15,16}$ that a long $\mathrm{Pu} / \mathrm{Py}$ sequence could selectively block transcription in one direction when assayed in in vitro transcription assays involving a bacteriophage RNA polymerase. Transcription of the DNA strand bearing the homopurine motif (leading to a transcript with a homopyrimidine motif) was observed in this experimental system, but no transcript originating from the opposite homopyrimidine DNA strand was produced. But in Escherichia coli, the presence of a homopyrimidine motif in the coding sequence (Py-rich mRNA transcribed from Pu-rich DNA strand) was shown to strongly reduce protein expression. ${ }^{17} \mathrm{~A}$ study conducted in COS-7 cells revealed that transcription of both DNA strands was reduced in living cells, mostly the transcription of the DNA strand bearing the homopyrimidine motif, but that protein expression was affected only in this latter case (when a Pu-rich mRNA was produced from the Py-rich DNA strand). ${ }^{18}$ Obviously, the existing 
differences between assays conducted in in vitro transcription systems or in living cells, and between bacterial and mammalian cells transcription machineries, might explain the observed discordances. However, additional investigations are clearly needed in order to expand our knowledge about the effect of the presence of a Pu/Py DNA sequence on transcription in living cells.

The thyroglobulin ( $\mathrm{Tg}$ ) gene, which encodes a key protein involved in thyroid hormone synthesis, is flanked on its proximal $5^{\prime}$ side by a $\mathrm{Pu} / \mathrm{Py}$ DNA element of variable length and primary sequence depending on the species. ${ }^{13}$ No role was found for this motif in the functional study of the Tg gene promoter region, as assayed by transient transfection of reporter DNA constructs into thyroid cells. ${ }^{19}$ We show here that this long $\mathrm{Pu} / \mathrm{Py}$ motif inhibits transcription in rat thyroid cells, both in its natural context or when placed next to a heterologous promoter. Contrary to the previous in vitro observations, ${ }^{3,15,16}$ transcription of both DNA strands was reduced.

\section{Materials and methods DNA constructions}

Oligonucleotides used for polymerase chain reaction (PCR) and DNA sequencing were purchased from Eurogentec (Seraing, Belgium). The sequences are shown in Table 1. All PCR-amplified sequences were verified by DNA sequencing in order to exclude the presence of sequence changes introduced during the PCR step.

\section{$\mathrm{Tg}$ reporter constructs}

The sequences of the $5^{\prime}$ end of the rat $\mathrm{Tg}$ gene extending from position -168 to +9 (relative to transcription start as +1 ) were amplified by PCR from PCCl3 thyroid cell genomic DNA using primers CC313 (forward) and CC118 (reverse), which added XhoI and Hind III restriction sites at $5^{\prime}$ and $3^{\prime}$ ends, respectively, and inserted between the corresponding cloning sites in the bidirectional reporter vector pGL4LUC-RLUC, ${ }^{20}$ yielding construct rTgLRL (Figure 1B).The $\mathrm{Pu} / \mathrm{Py}$ elements of rat and mouse Tg gene $5^{\prime}$ upstream regions were amplified by PCR from $\mathrm{PCCl} 3$ cell and mouse tail genomic DNAs, respectively, using the pairs of primers (forward + reverse) CC314 + CC315 and CC425 + CC426, respectively, which added NheI and EcoRI restrictions sites at the $5^{\prime}$ and $3^{\prime}$ ends of the amplicons, respectively, and inserted between the corresponding cloning sites in plasmid rTgLRL, giving constructs $\mathrm{Pu} /$ Py-rTgLRL (Figure 1B; Pu/Py is either rat or murine $\mathrm{Pu} / \mathrm{Py}$ sequence). For the construction of plasmid Py/Pu-rTgLRL
Table I Sequences of the oligonucleotides used as primers in PCR, qPCR, and RT-qPCR experiments

\begin{tabular}{|c|c|}
\hline Name & Sequence $\left(5^{\prime} \ldots . . . . .3^{\prime}\right)$ \\
\hline $\mathrm{CCII8}$ & CGAAGCTTCAGTGTCCCATCTGAGTCCC \\
\hline \multirow[t]{2}{*}{$\mathrm{CC} 313$} & CGCTCGAGCTCGAATTCACCCTACTGATTACTCAAG \\
\hline & TATTCTT \\
\hline $\mathrm{CC} 314$ & GCGCTAGCAATTTTCAAGAATAGTGTCTACAGCT \\
\hline CC3I5 & GCGAATTCTGCCAGAGCTTTGTACCCTT \\
\hline CC322 & GCCTCGAGCAGGTGTAAATGTGTGAATCAGA \\
\hline CC323 & GCAAGCTTCGCGCAGCGTTCACAAAG \\
\hline CC324 & GCAAGCTTCAGGTGTAAATGTGTGAATCAGA \\
\hline CC325 & GCCTCGAGCGCGCAGCGTTCACAAAG \\
\hline CC329 & GCGCTAGCAGTCAAGAGCTTGGTGGAACTT \\
\hline CC330 & GCGAATTCCCTGGACAATCAACCAGCAAAG \\
\hline \multirow[t]{2}{*}{ CC425 } & GCGCTAGCAATTTTCTACAGTATTACCTACAGA \\
\hline & TATTAGG \\
\hline CC426 & GCGAATTCTATAAAACTGTGGAAGATAAAATACCTC \\
\hline \multirow[t]{2}{*}{$\mathrm{CC} 427$} & GCGAATTCAATTTTCTACAGTATTACCTACAGA \\
\hline & TATTAGG \\
\hline CC428 & GCGCTAGCTATAAAACTGTGGAAGATAAAATACCTC \\
\hline CC430 & GCGGATCCTATAAAACTGTGGAAGATAAAATACCTC \\
\hline CC433 & GCGGATCCGCGCAGCGTTCACAAAG \\
\hline CC434 & GCGGATCCAGGTGTAAATGTGTGAATCAGA \\
\hline \multirow[t]{2}{*}{ CC435 } & GCCTCGAGATTTTCTACAGTATTACCTACAGATAT \\
\hline & TAGG \\
\hline CC439 & AGGACGCTCCAGATGAAAT \\
\hline CC444 & $(\mathrm{T})_{29}$ GTTGTTA \\
\hline CC445 & CAAGATCCGCGAGATTCTCATT \\
\hline CC446 & TTTCAAGAATAGTGTCTACAGCTGAATTGCTCTAA \\
\hline CC447 & GGGACAAGAAATGTGAGGCTAGCACTAA \\
\hline CC448 & AGGGGCATGTCGACCTTATGTGTAAA \\
\hline CC449 & TGCTTTAGAGCAATTCAGCTGTAGACACT \\
\hline CC450 & CTGCCAGCTACACAAGCAACGAA \\
\hline CC45I & СССTCTGTGTCTACACACCAGCATT \\
\hline
\end{tabular}

Abbreviations: PCR, polymerase chain reaction; $9 P C R$, quantitative PCR; RTqPCR, quantitative reverse transcription PCR.

(Figure $1 \mathrm{~B}$ ) in which the murine $\mathrm{Pu} / \mathrm{Py}$ sequence was placed in the reverse orientation relative to the $\mathrm{Tg}$ promoter, the same strategy as mentioned earlier was followed except that the pair of primers CC427 + CC428 was used in the PCR which resulted in the addition of EcoRI and NheI restrictions sites at the $5^{\prime}$ and $3^{\prime}$ ends of the amplicon, respectively. The sequence extending from -3469 to -3696 upstream of the rat $\mathrm{Tg}$ gene were amplified by PCR from $\mathrm{PCCl} 3$ cell genomic DNA, using primers CC329 (forward) and CC330 (reverse), which added NheI and EcoRI restriction sites in $5^{\prime}$ and $3^{\prime}$, respectively, and inserted between the corresponding cloning sites in plasmid rTgLRL, giving construct rDNArTgLRL (Figure 1B). For stable transformation of $\mathrm{PCCl} 3$ cells, the Renilla luciferase (RLUC) reporter gene and rat $\mathrm{Tg}$ promoter sequences with or without the $\mathrm{Pu} / \mathrm{Py}$ region were excised from plasmid Pu/Py-rTgLRL or rTgLRL, respectively, using Asp718 and HindIII, and inserted into (Asp718 + HindIII)-cleaved pGL4.17 vector (Promega Corp, Madison, WI, USA). 
A

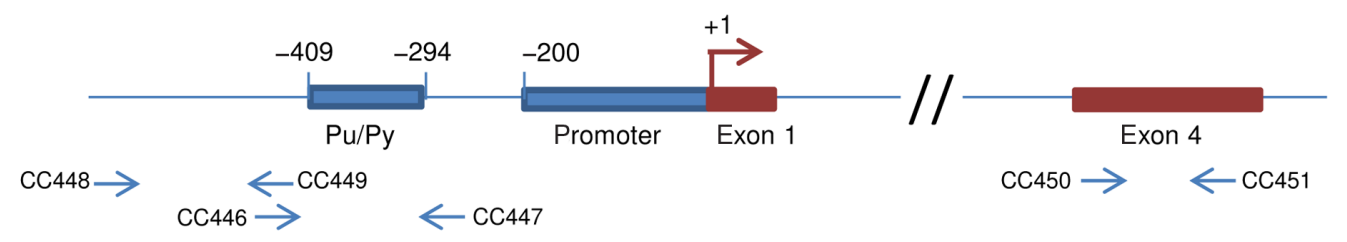

Rat Tg gene 5'-flanking sequence

TGCCATGGCTTCATTTTCAAGAATAGTGTCTACAGCTGAATTGCTCTAAAGCAATACTGAAAGAAGGAAGGAAGGAAAGAAGA AAGGAAGGATGAATGAAGCAAGGAAAAAAAGGAAAAGAAAGGGGACGAAGGAAGGAAGAAGAGGGGGAGGAATCAGGAGGA AGAGGAGATATTTTATCTTTCACAGTTTTACAATCGAACTGTCACCCCTAAGGGTACAAAGCTCTGGCATTTGCCTGTAAAGG GAAATTTTAGTGCTAGCCTCACATTTCTTGTCCCCATGTCCTGGAGTGGT CACCCTACTGATTACTCAAGTATTCTTAGCGGG AGCAGACTCAAGTAGAGGGAGTTCCTGTGACTAGCAGAGAAAACAAAGTGAGCCACTGCCCAGTCAAGTGTTCTTGAACAGTA GAGCACTGCTTGCCACTGTGCIATAAAGGCTTCCTGATAAGGGGACTCAGATGGGACACTGGTCCTACCCCATCATTTGAGTA

B
rTgLRL $+1$

rTgLRL

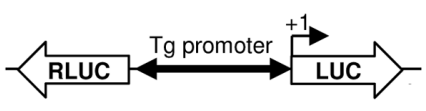

rTh2LRL
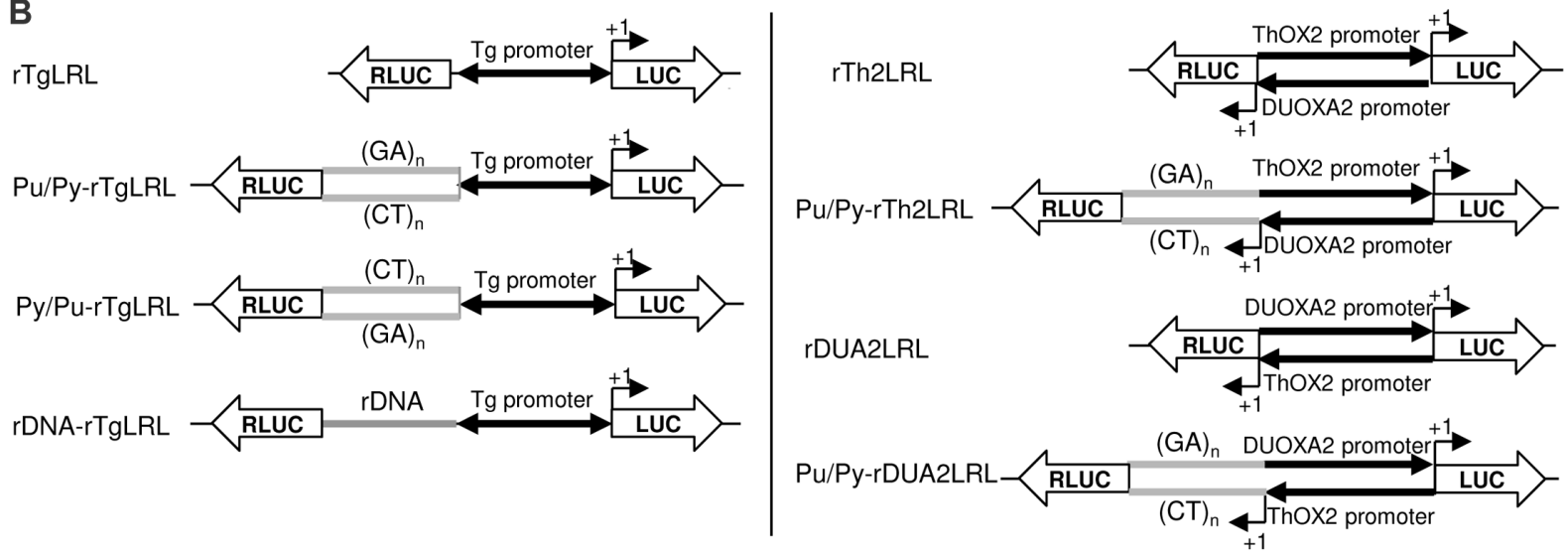

Figure I Genomic DNA sequences and reporter constructs used in the study.

Notes: (A) Schematic view of the organization of the $5^{\prime}$ end of the rat Tg gene with the locations of the primer pairs used in the (RT-)qPCR experiments, and sequence of the rat $\mathrm{Tg}$ gene 5 '-flanking region showing the homopurine-homopyrimidine (Pu/Py) region used in our reporter constructs (light gray shaded) and the Pu/Py sequence itself (bold characters), the CAT motifs (underlined; ATG on complementary strand: see text), and the Tg promoter region used in the reporter constructs (bold italics, double-underlined TATA box and TSS indicated as “+l”). (B) Schematic representation of the reporter constructs used in this study. Firefly luciferase (LUC) and Renilla luciferase (RLUC) genes are positioned in a head-to-head configuration. (GA) and (CT): Pu/Py region in forward and reverse orientation, respectively; rDNA: rat genomic DNA fragment (see text).

Abbreviations: Pu/Py, homopurine-homopyrimidine; PCR, polymerase chain reaction; qPCR, quantitative PCR; RT-qPCR, quantitative reverse transcription PCR; TSS, transcription start site.

\section{ThOX2-DUOXA2 reporter constructs}

The bidirectional promoter region of ThOX2 and DUOXA2 genes $^{20}$ was amplified by PCR from PCCl3 cell genomic DNA using the pairs of primers (forward + reverse) CC324 + $\mathrm{CC} 325$ or $\mathrm{CC} 322+\mathrm{CC} 323$ which added HindIII and XhoI restriction sites at $5^{\prime}$ and $3^{\prime}$ or $3^{\prime}$ and $5^{\prime}$ ends, respectively, of the $190 \mathrm{bp}$ long amplicon. The amplified sequences were inserted between the corresponding cloning sites in the bidirectional reporter vector pGL4LUC-RLUC, yielding construct rTh2LRL or rDUA2LRL, respectively (Figure 1B). The $\mathrm{Pu} / \mathrm{Py}$ element of the mouse Tg gene $5^{\prime}$ upstream region was amplified by PCR from mouse tail genomic DNA using primers CC435 (forward) and CC430 (reverse), which added XhoI and BamHI restriction at $5^{\prime}$ and $3^{\prime}$ ends, respectively, and the bidirectional promoter region of ThOX2 and DUOXA2 genes was amplified by PCR from $\mathrm{PCCl} 3$ cell genomic DNA using the pairs of primers (forward + reverse) $\mathrm{CC} 324+\mathrm{CC} 433$ or
$\mathrm{CC} 434+\mathrm{CC} 323$ which added HindIII and BamHI restriction sites at $5^{\prime}$ and $3^{\prime}$ or $3^{\prime}$ and $5^{\prime}$ ends, respectively. The amplified $\mathrm{Pu} / \mathrm{Py}$ and promoter sequences were inserted together into (XhoI + HindIII)-cleaved bidirectional reporter vector pGL4LUC-RLUC, yielding construct Pu/Py-rTh2LRL or $\mathrm{Pu} / \mathrm{Py}-\mathrm{rDUA} 2 \mathrm{LRL}$, respectively (Figure 1B).

\section{Cell culture and DNA transfections}

PCCl3 cells were cultured as described by Fusco and coworkers. ${ }^{21}$ The cells were trypsinized and seeded at $30 \%-40 \%$ confluence the day preceding transfection. Cotransfections were performed using $3.3 \mu \mathrm{L}$ of FuGENE 6 transfection reagent (Roche Diagnostics $\mathrm{GmbH}$, Mannheim, Germany) and $1.1 \mu \mathrm{g}$ of DNA per dish (diameter: $3.5 \mathrm{~cm}$ ). To each individual cell culture dish were added $1 \mu \mathrm{g}$ of firefly (LUC) + Renilla (RLUC) luciferases reporter construct and $100 \mathrm{ng}$ of pTPO-SEAP, ${ }^{22}$ containing the secreted alkaline 
phosphatase coding sequence under the control of the human thyroperoxidase gene promoter and used as internal control for normalization in regard to transfection efficiency. In order to obtain $\mathrm{PCCl} 3$ cell clones containing copies of the reporter construct integrated within their genome, transfection was performed by adding to the dish (diameter: $6 \mathrm{~cm}$ ) $1 \mu \mathrm{g}$ of the reporter plasmid (pGL4.17 vector backbone) and FuGENE 6 transfection reagent at a ratio of FuGENE $6(\mu \mathrm{L})$ to DNA $(\mu \mathrm{g})$ of 3:1. After 24 hours, cells stably transformed with the reporter construct were selected in culture medium supplemented with $400 \mu \mathrm{g} / \mathrm{mL}$ of Geneticin (Thermo Fisher Scientific, Waltham, MA, USA) and maintained afterward in the presence of $200 \mu \mathrm{g} / \mathrm{mL}$ of Geneticin.

\section{Luciferase assays}

LUC and RLUC activities were measured between 24 and 48 hours after transfection using the Dual-Luciferase ${ }^{\circledR}$ Reporter Assay System as recommended by the manufacturer (Promega Corp, Madison, WI, USA) and a TD-20/20 luminometer (Turner Designs, Sunnyvale, CA, USA). Secreted embryonic alkaline phosphatase (SEAP) activity present in the culture medium was measured at the same time using the PhosphaLight $^{\text {TM }}$ System (Tropix Inc. [Thermo Fisher], Bedford, MA, USA). All transfections were performed in duplicate in each individual experiment. LUC and RLUC activities were expressed in arbitrary units as displayed on the luminometer after a 10 -second integration time and normalized against SEAP production in the individual transfected dishes. The RLUC/LUC ratio was arbitrarily set to the value of one for the construct containing only the promoter sequence. Statistical analysis of the data was performed using the two-tailed MannWhitney test available on the VassarStats web site for statistical computation (http://vassarstats.net/index.html).

\section{qPCR and RT-qPCR experiments}

Total RNA was obtained from cultured PCCl3 cells (dish diameter: $10 \mathrm{~cm}$ ) using the RNeasy Mini Kit from Invitrogen (Life Technologies Europe BV, Gent, Belgium). Residual DNA was removed from the total RNA preparations using the Turbo DNA-free kit (Thermo Fisher Scientific). RNA was reverse transcribed into cDNA by random priming using the Superscript IIRT system from Invitrogen. RNA from PCCl3 cells transiently transfected with a reporter construct was obtained identically but reverse transcribed using an oligodT primer $\left(\mathrm{T}_{15}\right)$. Genomic DNA was obtained from PCCl3 cells using a standard protocol. ${ }^{23}$ For the analysis of natural transcripts qPCR (quantitative PCR) or RT-qPCR (quantitative reverse transcription PCR) mixes contained $10 \mathrm{ng}$ of genomic DNA or $6.25 \mathrm{ng}$ of cDNA, respectively, and $200 \mathrm{nM}$ of each primer (Table 2) in the $\mathrm{iQ}^{\mathrm{TM}} \mathrm{SYBR}$ Green Supermix (Bio-Rad Laboratories, Hercules, CA, USA). Reactions (final volume: $20 \mu \mathrm{L}$ ) were performed in a C1000 ${ }^{\mathrm{TM}}$ Thermal Cycler equipped with a CFX96 ${ }^{\mathrm{TM}}$ Real-Time System (BioRad Laboratories) using an initial activation step $\left(95^{\circ} \mathrm{C}\right.$, 3 minutes) and 40 repetitions of a two-step cycle, first step at $95^{\circ} \mathrm{C}$ for 10 seconds and second step at $60^{\circ} \mathrm{C}$ for 1 minute, and were followed by a melting curve analysis of the produced amplicons. For the quantification of LUC and RLUC transcripts, 25 ng of cDNA was used with identical primer concentrations, basal reaction mix, and initial activation step, and then 40 repetitions of a two-step cycle, first step at $95^{\circ} \mathrm{C}$ for 10 seconds and second step at $56^{\circ} \mathrm{C}$ for 1 minute, were performed and followed by a melting curve analysis of the produced amplicons. The primer pairs (forward + reverse) used were as follows: LUC: CC445 + CC444 (amplicon size: 255 bp); RLUC: CC439 + CC 444 (amplicon size: 268 bp). Reactions were done in duplicate in each experiment and the experiments were repeated at least once.

\section{Results and discussion}

\section{The 5' homopurine-homopyrimidine DNA sequence of the thyroglobulin gene reduces reporter gene expression}

The Tg gene promoter is flanked on its $5^{\prime}$ side by a long $\mathrm{Pu} /$ Py DNA sequence in several mammalian species (Figure 1A), including man. ${ }^{13}$ This classical TATA box-containing promoter directs the unidirectional production of Tg mRNA in thyroid cells. However, by inserting the minimal Tg gene promoter region in a bidirectional reporter plasmid harboring both the LUC and the RLUC genes in a head-to-head configuration

Table 2 Absence of stable antisense transcripts originating from the $\mathrm{Tg}$ promoter in rat thyroid cells

\begin{tabular}{llll}
\hline $\mathbf{I}$ & $\mathbf{2}$ & $\mathbf{3}$ & $\mathbf{4}$ \\
$\begin{array}{l}\text { Target } \\
\text { sequence }\end{array}$ & $\begin{array}{l}\text { Cq value of } \\
\text { qPCR } \\
\text { on genomic DNA }\end{array}$ & $\begin{array}{l}\text { Cq value of } \\
\text { RT-qPCR } \\
\text { on total RNA }\end{array}$ & $\begin{array}{l}\text { Cq value of } \\
\text { qPCR } \\
\text { on total RNA }\end{array}$ \\
\hline $\begin{array}{l}\text { Upstream of } \\
\text { Pu/Py }\end{array}$ & $26.72 \pm 0.2$ & $37.5 \pm 1.5$ & $38.5 \pm 0.3$ \\
$\begin{array}{l}\text { Pu/Py region } \\
\text { Exon } 4 \text { of }\end{array}$ & $26.93 \pm 0.18$ & $38.5 \pm 0.5$ & Not detected \\
Tg gene & $25.92 \pm 0.05$ & $19.06 \pm 0.02$ & Not detected \\
\hline
\end{tabular}

Notes: $\mathrm{Cq}$ values (mean of duplicates \pm half-range) obtained in representative qPCR (columns 2 and 4) and RT-qPCR (column 3) experiments involving genomic DNA (column 2) or total RNA (columns 3 and 4) are shown. The pairs of primers used were as follows: upstream of Pu/Py: CC448 + CC449; Pu/Py region: CC446 + CC447; exon 4 of Tg gene: CC450 + CC45I.

Abbreviations: Pu/Py, homopurine-homopyrimidine; PCR, polymerase chain reaction; qPCR, quantitative PCR; RT-qPCR, quantitative reverse transcription PCR. 
(see construct rTgLRL in Figure 1B), we observed an efficient expression of both reporter genes, presumably as a consequence of the production of promoter upstream transcripts (PROMPTs) with appropriate stabilization signals by the antisense transcriptional activity of the promoter. ${ }^{24}$ When inserting the $\mathrm{Pu} / \mathrm{Py}$ sequence in its natural upstream position relative to the $\mathrm{Tg}$ promoter in the bidirectional reporter construct (see construct $\mathrm{Pu} / \mathrm{Py}$-rTgLRL in Figure 1B), we took care not to include potential translation initiation codons as they would interfere with RLUC expression by initiating translation upstream from the RLUC ORF (those codons appearing as CAT on the top strand in Figure 1A are underlined). As a control, we also constructed another plasmid in which a fragment of intergenic DNA of similar size (and also devoid of potential translation initiation codons), corresponding to the sequences located between $-3,469$ and $-3,696$ relative to the $\mathrm{Tg}$ gene TSS, was inserted at the same position relative to the $\mathrm{Tg}$ promoter (see construct rDNA-rTgLRL in Figure 1B). This construct would allow us to confirm that any observed effect with the $\mathrm{Pu} / \mathrm{Py}$-containing construct was actually related to its $\mathrm{Pu} / \mathrm{Py}$ content and not only to the size of the insert. The choice of using bidirectional reporter constructs was dictated by the ability of the studied $\mathrm{Pu} / \mathrm{Py}$ element to adopt a triplex structure (H-DNA) when present on supercoiled plasmid DNA $^{13}$, which could possibly affect the transfection efficiency of the plasmids bearing this element as compared to the control plasmids devoid of the $\mathrm{Pu} / \mathrm{Py} \mathrm{DNA}$ sequence. As previous work had demonstrated that the presence of the $\mathrm{Pu} / \mathrm{Py}$ motif did not influence Tg promoter activity, ${ }^{19}$ the expression of the LUC reporter gene could serve as a plasmid-specific internal control for transfection efficiency of the different reporter constructs used. Indeed, as shown in Figure 2A, substantial differences were observed in the level of LUC expression from the different reporter constructs even after normalization for overall transfection efficiency by using a co-transfected reporter construct encoding SEAP. The effect of the presence of the $\mathrm{Pu} / \mathrm{Py}$ element on the expression of the RLUC reporter gene was thus evaluated by calculating the ratio of RLUC expression relative to LUC expression for each of the individual reporter plasmids. An arbitrary ratio of 1 was set for the parental reporter construct containing only the promoter region. As shown in Figure 2B, after transfection into $\mathrm{PCCl} 3$ thyroid cells, the construct containing the $\mathrm{Pu} / \mathrm{Py}$ element in its natural upstream location relative to the Tg promoter (rPu/Py-rTgLRL) displayed a substantially decreased RLUC/LUC expression ratio as compared to both parent (Tg promoter alone, rTgLRL) and control DNA insert (rDNA-rTgLRL) constructs.
The murine $\mathrm{Pu} / \mathrm{Py}$ sequence homologous to that of the rat $\mathrm{Tg}$ gene promoter displays only limited primary sequence conservation with its counterpart in the rat $(72 \%$ of base identity; random $\mathrm{Pu} / \mathrm{Py}$ sequences scoring $50 \%$ base identity by chance only). Replacing the rat $\mathrm{Pu} / \mathrm{Py}$ element by its murine counterpart resulted in a very similar reduction in antisense gene expression (Figure 2B; construct mPu/Py-rTgLRL), which supported the view that the $\mathrm{Pu} / \mathrm{Py}$ asymmetry rather than the primary DNA sequence itself was responsible for the observed effect on gene expression.

To investigate whether the effect on gene expression was dependent on the orientation of the $\mathrm{Pu} / \mathrm{Py}$ sequence relative to the promoter, we also placed the same $\mathrm{Pu} / \mathrm{Py}$ element in the opposite orientation relative to the Tg promoter in construct Py/Pu-rTgLRL (Figure 1B). As shown in Figure 2B (construct $\mathrm{mPy} / \mathrm{Pu}-\mathrm{rTgLRL}), \mathrm{RLUC}$ expression was also decreased in this case, but to a lesser extent than when the $\mathrm{Pu} / \mathrm{Py}$ motif was positioned in the other orientation. However, it must be noted that when comparing the levels of RLUC expression from $\mathrm{Pu} / \mathrm{Py}$ and $\mathrm{mPy} / \mathrm{Pu}-\mathrm{rTgLRL}$ constructs normalized against the co-expressed SEAP reporter (Figure 2A), the difference is lower than when comparing the ratio of RLUC to LUC expression from the same constructs (Figure 2B) due to the decreased LUC expression from mPy/Pu-rTgLRL construct as compared to $\mathrm{mPu} / \mathrm{Py}-\mathrm{rTgLRL}$ (Figure 2A). Obviously, the effect of the $\mathrm{Pu} / \mathrm{Py}$ element on gene expression was not strictly orientation-dependent, contrary to what was observed previously in vitro..$^{3,15,16}$ In addition, in our experiments the $\mathrm{Pu} / \mathrm{Py}$ element exerted its main effect on the opposite transcriptional direction as compared to what had been observed in these in vitro experiments. According to these previous reports, the production of RLUC reporter transcripts containing a homopyrimidine RNA motif from the constructs $\mathrm{Pu} /$ Py-rTgLRL, which leads to the lower productions of RLUC in our experiments, should not be inhibited. In COS-7 cells transcription of both DNA strands was also reduced, in agreement with our observations on reporter gene expression in rat thyroid cells, but protein expression was not affected accordingly. ${ }^{18}$ It is conceivable that cell-specific factors may be involved in these distinct outcomes.

We also investigated whether the same effect could be observed when transcription was driven by other promoters. To do this, the $\mathrm{Pu} / \mathrm{Py}$ element was inserted on either side of the bidirectional ThOX2-DUOXA2 promoter region ${ }^{20}$ (see constructs $\mathrm{Pu} / \mathrm{Py}-\mathrm{rTh}$ 2LRL and $\mathrm{Pu} / \mathrm{Py}$-rDUA2LRL in Figure 1B) and the expression of the reporter genes driven by these constructs were compared to those obtained with the parent plasmids (constructs rTh2LRL and rDUA2LRL, 


\section{A Relative LUC and RLUC activities}

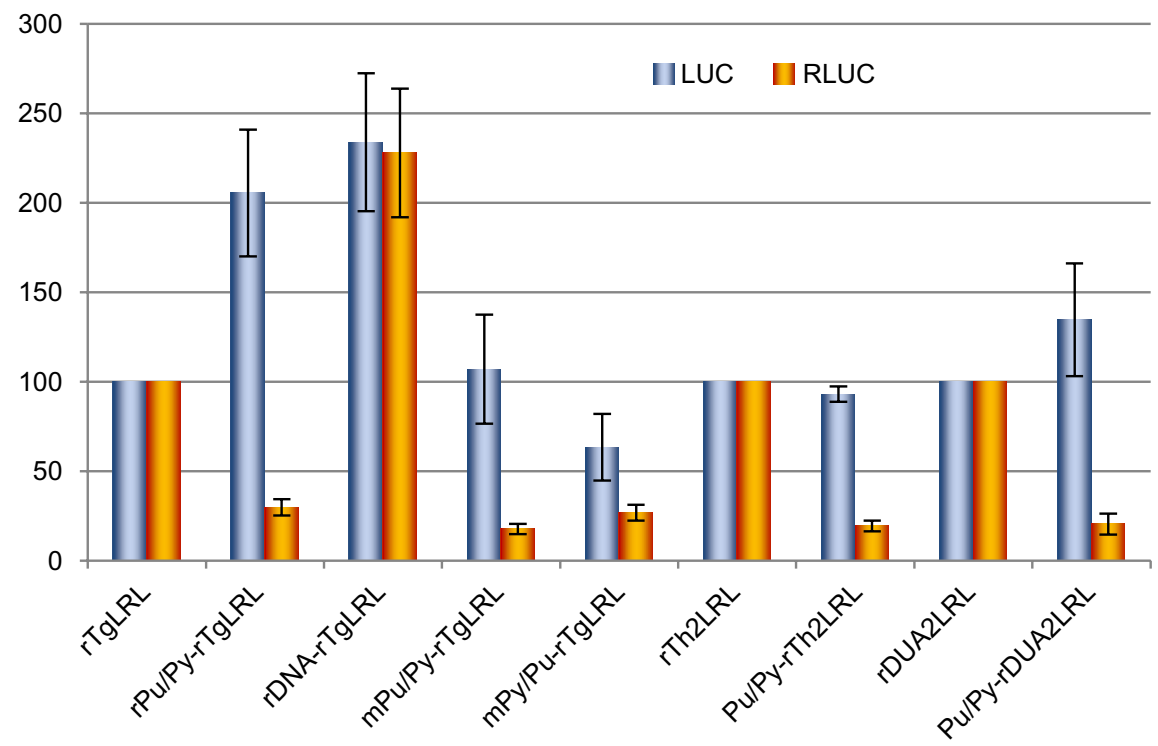

\section{B RLUC/LUC ratio (arbitrary units)}

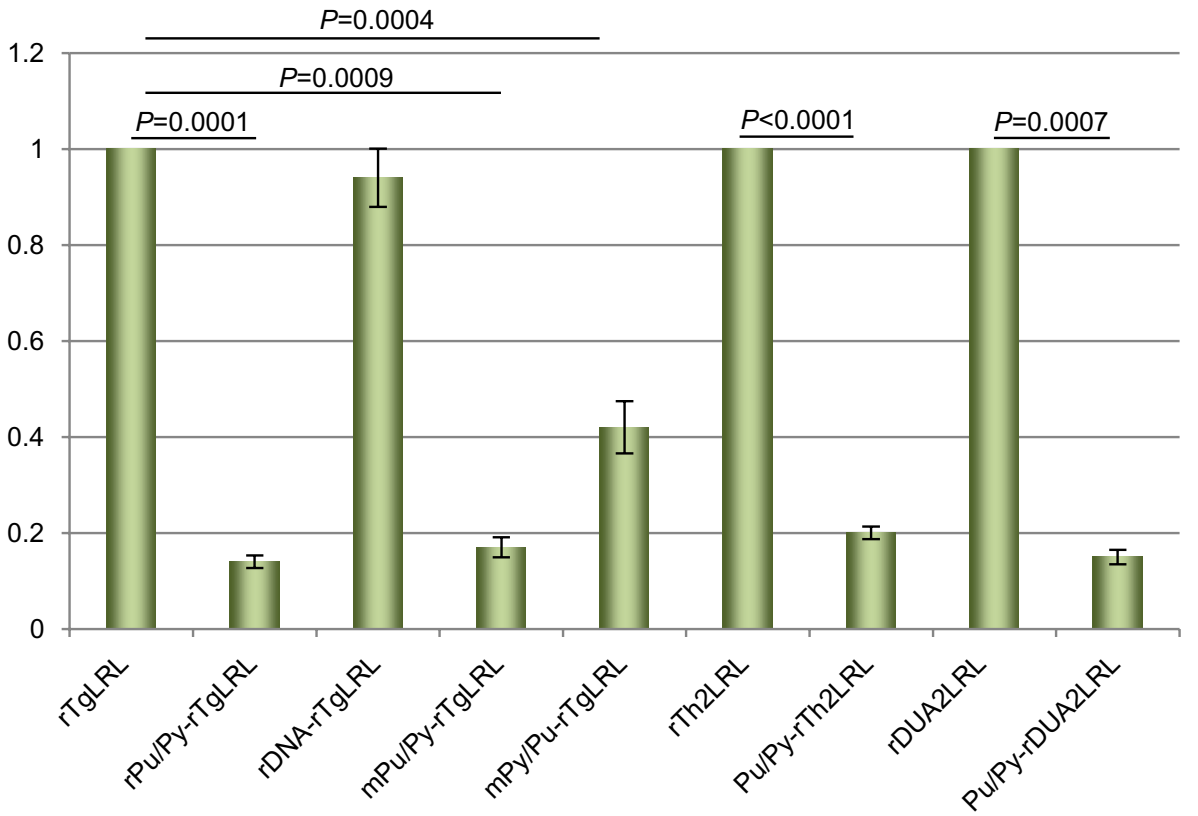

Figure 2 The presence of the Pu/Py element reduces reporter gene expression.

Notes: (A) The reporter constructs (see Figure IB for detailed structures) were transiently transfected in rat thyroid PCCI3 cells and the expressions of LUC and RLUC were measured individually in the same transfected cells. The LUC and RLUC values were arbitrarily set to I00\% for the construct containing only the promoter region. (B) The same results were expressed as ratios of RLUC/LUC production for each of the constructs. The ratio was arbitrarily set to I for the construct containing only the promoter region. The results shown are mean \pm SD from at least three independent experiments. Statistical analysis was performed using two-tailed Mann-Whitney test. Abbreviations: Pu/Py, homopurine-homopyrimidine; LUC, firefly luciferase; RLUC, Renilla luciferase; SD, standard deviation.

respectively, in Figure 1B). As shown in Figure 2B, the presence of the $\mathrm{Pu} / \mathrm{Py}$ sequence decreased the relative level of RLUC expression driven by either the ThOX2 promoter or the DUOXA2 promoter. Thus, the $\mathrm{Pu} / \mathrm{Py}$ element of the Tg promoter inhibited gene expression from both of these promoters as well as antisense gene expression from the $\mathrm{Tg}$ promoter itself.

\section{The homopurine-homopyrimidine DNA motif negatively controls mRNA}

\section{accumulation}

Before it could be concluded that the presence of the $\mathrm{Pu} / \mathrm{Py}$ element reduced transcriptional activity in our transfections, we had to exclude the possibility that the presence of the $\mathrm{Pu} /$ Py sequence in the $5^{\prime}$ UTR (untranslated region) of the mRNA 
prevented efficient translation of the RLUC ORF. A decreased RLUC production could indeed result either from a lower amount of RLUC mRNA or from a reduced translational efficiency of this mRNA. We thus conducted RT-qPCR experiments on mRNA preparations from transfected cells with the aim of quantifying both LUC and RLUC transcripts. The relative amounts of LUC and RLUC mRNAs were measured in RNA preparations from cells transfected with the Tg promoter reporter constructs containing or not the $\mathrm{Pu} / \mathrm{Py}$ element. We devised a specific strategy relying on the use of an anchored oligo-dT primer in the RT-qPCR experiments in order to prevent the amplification of non-polyadenylated LUC or RLUC coding sequence. The absence of amplification when reverse transcription of the RNAs was omitted confirmed the specificity of our measure. The results of the RT-qPCR experiments were expressed as $2^{\text {(Cq RLuc-CqLuc) }}$ and this value was arbitrarily set to 1 in the case of the $\mathrm{Pu} / \mathrm{Py}$-less reporter construct. As depicted in Figure 3, the value of $2^{\triangle \mathrm{Cq}}$ was higher for the $\mathrm{Pu} / \mathrm{Py}$-containing construct, indicating that the RLUC/LUC mRNA ratio was significantly decreased in RNA preparations from cells transfected with the $\mathrm{Pu} / \mathrm{Py}$-containing reporter construct as compared to the $\mathrm{Pu} / \mathrm{Py}$-less reporter transfected cells (the higher $2^{\Delta \mathrm{Cq}}$ value reflected the increased $\mathrm{Cq}$ value obtained for RLUC compared with LUC cDNAs, which corresponded to a decreased amount of RLUC cDNA compared with LUC cDNA). Thus, the accumulation of transcripts originating from the Tg promoter was clearly reduced on the strand bearing the $\mathrm{Pu} / \mathrm{Py}$ sequence as compared to the opposite direction.

We also investigated if PROMPTs originating from the $\mathrm{Tg}$ promoter and encompassing the $\mathrm{Pu} / \mathrm{Py}$ sequence

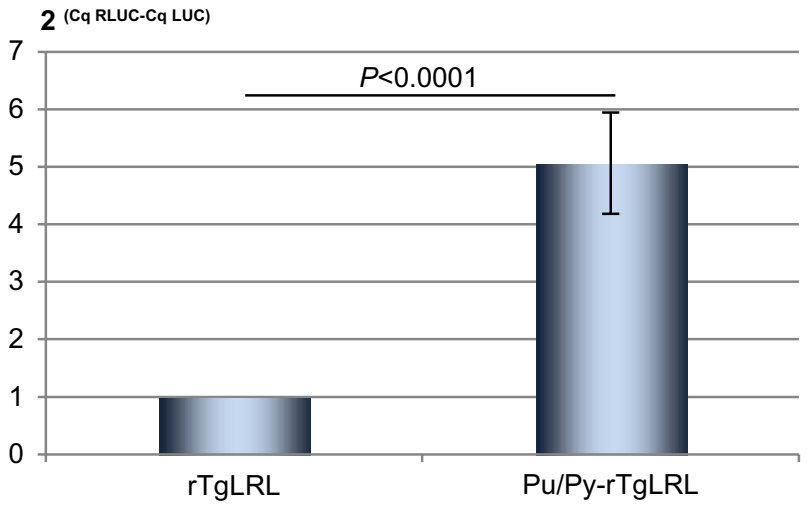

Figure 3 LUC and RLUC mRNAs levels in cells transfected with Pu/Py-containing or Pu/Py-less reporter construct as determined by RT-qPCR.

Notes: The results shown are the means \pm SD from six individual measures expressed in the form of $2^{\Delta C_{q}}$ relatively to the value obtained for the Pu/Py-less reporter construct which was arbitrarily set to I. Statistical analysis was performed using two-tailed Mann-Whitney test.

Abbreviations: Pu/Py, homopurine-homopyrimidine; LUC, firefly luciferase; RLUC, Renilla luciferase; RT-qPCR, quantitative reverse transcription polymerase chain reaction; SD, standard deviation. accumulated to a detectable level in rat thyroid cells. RT-qPCR experiments were performed on total RNA. We used two primer pairs directing the amplification of sequences located upstream from the promoter and a pair of primers enabling the amplification of sequences in exon 4 of the Tg gene as a positive control (Figure 1A). When assessed in qPCR experiments using genomic DNA, all three primers pairs directed the amplification of their target sequences with comparable efficiencies (Table 2, column 2). By contrast, only the sequences of exon 4 were significantly amplified in the RT-qPCR experiments (Table 2, column 3), indicating the absence of significant amounts of transcripts encompassing the sequences located upstream from the promoter in the RNA preparations. Omitting the reverse transcription step resulted in the absence of significant amplification in all three cases as expected (Table 2, column 4). Thus, no significant level of PROMPTs encompassing the upstream Pu/Py motif of the Tg gene could be detected in rat thyroid cells, which is in agreement with the adverse effect of this DNA sequence on transcription observed in our previous experiments. Whether limiting the extension of PROMPTs originating from the $\mathrm{Tg}$ promoter constitutes the main physiological function of the evolutionary conserved $\mathrm{Pu} / \mathrm{Py}$ element lying in the $5^{\prime}$ vicinity of the Tg promoter remains, however, to be investigated specifically.

\section{The transcription-blocking effect also occurs in a genomic context}

When present on supercoiled plasmid DNA, the $\mathrm{Pu} / \mathrm{Py}$ sequence we used could adopt a triple-helical structure called H-DNA. ${ }^{13,14}$ We thus investigated whether the effect we observed using transiently transfected plasmid DNAs could be reproduced when the DNA constructs were integrated into the cellular genomic DNA. Cell clones stably transformed with either the $\mathrm{Pu} / \mathrm{Py}$-containing or the $\mathrm{Pu} / \mathrm{Py}$-less Tg promoter reporter construct were established and the relative amounts of LUC and RLUC activity present in pools of these clones were quantified. As shown in Figure 4, the expected difference in the mean ratio of RLUC/LUC expression was observed between pools of cell clones transformed with the $\mathrm{Pu} / \mathrm{Py}$-containing or the $\mathrm{Pu} / \mathrm{Py}$-less reporter construct. Thus, the presence of the $\mathrm{Pu} / \mathrm{Py}$ region reduced gene expression from the Tg promoter in a genomic context as well as when assayed using transfected plasmids.

\section{Conclusion}

Contrary to previous observations made in vitro using a bacteriophage RNA polymerase, ${ }^{3,15,16} \mathrm{Pu} / \mathrm{Py} \mathrm{DNA}$ sequences do 


\section{RLUC/LUC ratio (arbitrary units)}

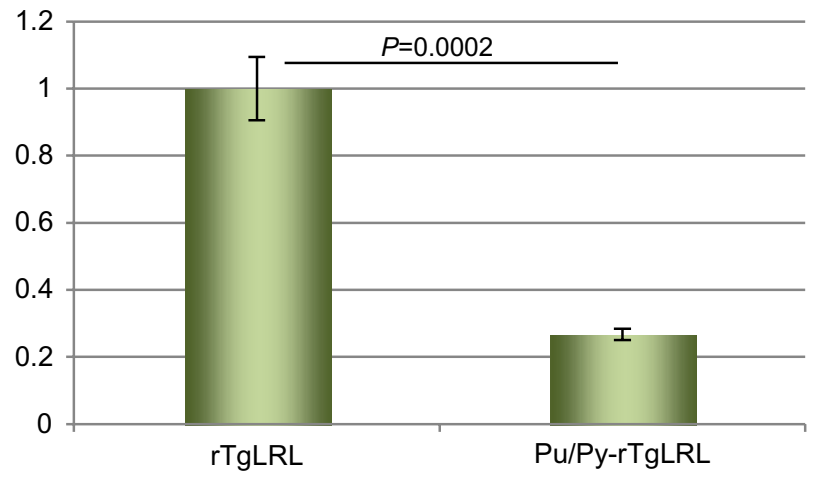

Figure 4 Assay of the transcription-blocking effect of the Pu/Py sequence in a genomic context.

Notes: Ratios of RLUC/LUC expression in pools of clones of rat thyroid $\mathrm{PCCl} 3$ cells transformed with $\mathrm{Pu} / \mathrm{Py}$-containing or Pu/Py-less $\mathrm{Tg}$ promoter reporter construct are shown. The ratio was arbitrarily set to I for the construct containing only the promoter region. Four individual measures of RLUC/LUC ratio in independent pools of transformed cells were made for each construct. Statistical analysis was performed using two-tailed Mann-Whitney test.

Abbreviations: LUC, firefly luciferase; RLUC, Renilla luciferase; Pu/Py, homopurinehomopyrimidine.

not appear to behave as transcriptional diodes in mammalian cells. In COS-7 cells ${ }^{18}$ and in rat thyroid cells (this study) at least, their presence reduced transcriptional activity on both DNA strands, with some minor differences in the level of transcription of one DNA strand relative to the other depending on the cell type.

\section{Acknowledgments}

We are grateful to Dr S Costagliola (Université Libre de Bruxelles, IRIBHM, campus Erasme, 1070 Bruxelles, Belgique) for providing us with the pTPO-SEAP construct and to Dr M Ludgate (Cardiff University, School of Medicine, United Kingdom) for careful reading of the paper. This work was supported by grants from the Belgian Fonds National de la Recherche Scientifique Médicale (FRSM, grant 3.4550.09), the Communauté Française de Belgique - Actions de Recherche Concertées (ARC), the Belgian program "Pôles d'Attraction Interuniversitaire" (PAI, Prime Minister's Office, Science Policy Programming), and the Belgian Fonds David et Alice Van Buuren.

\section{Disclosure}

DC is a research director of the Belgian Fonds National de la Recherche Scientifique (FNRS). The authors report no other conflicts of interest in this work.

\section{References}

1. Albrecht-Buehler G. Properties and distribution of pure GA-sequences of mammalian genomes. PLoS ONE. 2008;3(11):e3818.
2. Lu G, Ferl RJ. Homopurine/homopyrimidine sequences as potential regulatory elements in eukaryotic cells. Int $J$ Biochem. 1993;25:1529-1537.

3. Grabczyk E, Fishman MC. A long purine-pyrimidine homopolymer acts as a transcriptional diode. J Biol Chem. 1995;270:1791-1797.

4. Glaser RL, Thomas GH, Siegfried E, Elgin SCR, Lis JT. Optimal heat-induced expression of the Drosophila hsp26 gene requires a promoter sequence containing (CT) -(GA), repeats. J Mol Biol. 1990;211: 751-761.

5. Raghu G, Tevosian S, Anant S, Subramanian KN, George DL, Mirkin SM. Transcriptional activity of the homopurine-homopyrimidine repeat of the c-Ki-ras promoter is independent of its $\mathrm{H}$-forming potential. Nucl Acids Res. 1994;22:3271-3279.

6. Darvish H, Nabi MO, Firouzabadi SG, et al. Exceptional human core promoter nucleotide compositions. Gene. 2011;475:79-86.

7. Choi HS, Hwang CK, Song KY, Law P-Y, Wei L-N, Loh HH. Poly(C)binding proteins as transcriptional regulators of gene expression. Biochem Biophys Res Commun. 2009;380:431-436.

8. Rao BS. Regulation of DNA replication by homopurine/homopyrimidine sequences. Mol Cell Biochem. 1996;156:163-168.

9. Krasilnikov AS, Panyutin IG, Samadashwily GM, Cox R, Lazurkin YS, Mirkin SM. Mechanisms of triplex-caused polymerization arrest. Nucl Acids Res. 1997;25:1339-1346.

10. Krasilnikova MM, Smirnova EV, Krasilnikov AS, Mirkin SM. A new trick for an old dog: TraY binding to a homopurine-homopyrimidine run attenuates DNA replication. J Mol Biol. 2001;313:271-282.

11. Kohwi Y, Panchenko Y. Transcription-dependent recombination induced by triple-helix formation. Gene Dev. 1993;7:1766-1778.

12. Bacolla A, Collins JR, Gold B, et al. Long homopurine-homopyrimidine sequences are characteristic of genes expressed in brain and the pseudoautosomal region. Nucl Acids Res. 2006;34:2663-2675.

13. Christophe D, Cabrer B, Bacolla A, Targovnik H, Pohl V, Vassart G. An unusually long poly(purine)-poly(pyrimidine) sequence is located upstream from the human thyroglobulin gene. Nucl Acids Res. 1985; 13:5127-5144.

14. Htun H, Dahlberg JE. Topology and formation of triple-stranded H-DNA. Science. 1989;243:1571-1576.

15. Belotserkovskii BP, Liu R, Tornaletti S, Krasilnikova MM, Mirkin SM, Hanawalt PC. Mechanisms and implications of transcription blockage by guanine-rich DNA sequences. Proc Natl Acad Sci U SA. 2010;107: 12816-12821.

16. Belotserkovskii BP, Neil AJ, Saleh SS, Shin JH, Mirkin SM, Hanawalt PC. Transcription blockage by homopurine DNA sequences: role of sequence composition and single-strand breaks. Nucl Acids Res. 2013;41:1817-1828.

17. Sarkar PS, Brahmachari SK. Intramolecular triplex potential sequence within a gene down regulates its expression in vivo. Nucl Acids Res. 1992;20:5713-5718.

18. Ohshima K, Montermini L, Wells RD, Pandolfo M. Inhibitory effect of expanded GAA-TTC triplet repeats from intron 1 of the Friedreich ataxia gene on transcription and replication in vivo. $J$ Biol Chem. 1998;273:14588-14595.

19. Christophe D, Gérard C, Juvénal G, et al. Identification of a cAMPresponsive region in thyroglobulin gene promoter. Mol Cell Endocrinol. 1989;64:5-18.

20. Christophe-Hobertus C, Christophe D. Delimitation and functional characterization of the bidirectional THOX-DUOXA promoter regions in thyrocytes. Mol Cell Endocrinol. 2010;317:161-167.

21. Fusco A, Berlingieri MT, Di Fiore PP, Portella G, Grieco M, Vecchio G. One- and two-step transformations of rat thyroid epithelial cells by retroviral oncogenes. Mol Cell Biol. 1987;7:3365-3370.

22. Vilain C, Rydlewski C, Duprez L, et al. Autosomal dominant transmission of congenital thyroid hypoplasia due to loss-of-function mutation of PAX8. J Clin Endocrinol Metab. 2001;86:234-238.

23. Sambrook J, Russell DW. Molecular Cloning: A Laboratory Manual. Cold Spring Harbor, NY: Cold Spring Harbor Laboratory Press; 2001.

24. Ntini E, Järvelin AI, Bornholdt J, et al. Polyadenylation site-induced decay of upstream transcripts enforces promoter directionality. Nat Struct Mol Biol. 2013;20:923-928. 
Research and Reports in Biology

\section{Publish your work in this journal}

Research and Reports in Biology is an international, peer-reviewed, open access journal publishing original research, reports, editorials, reviews and commentaries on all areas of biology including animal biology, biochemical biology, cell biology, ecological studies, evolutionary biology, molecular biology, plant science and botany. The

manuscript management system is completely online and includes a very quick and fair peer-review system. Visit http://www.dovepress com/testimonials.php to read real quotes from published authors.

Submit your manuscript here: http://www.dovepress.com/research-and-reports-in-biology-journal 